\title{
ACO based Clustering Protocol for Resource Constrained Mobile Adhoc Networks
}

\author{
Shilpa Suresh \\ Dept. of Electronics and Communication \\ Hindustan Institute of Technology and Science \\ Chennai, India
}

\author{
Aby $\mathrm{K}$ Thomas \\ Dept. of Electronics and Communication \\ Hindustan Institute of Technology and Science \\ Chennai, India
}

\begin{abstract}
Mobile Ad-hoc networks (MANETs) are increasingly popular with recent advancements in computer and communications technologies. A mobility-based clustering (MBC) protocol for wireless ad hoc network with mobile nodes works well in an energy constrained network environment, where a node elects itself as a cluster-head based on its residual energy and mobility. A non-cluster-head node aims at its link stability with a cluster head during its association in the estimated connection time. In a centralized network, data packets from source need to travel to the destination with minimum number of hops, which commendably reduces the energy requirement. Still there exist chances for congestion occurrence in course of the transmission if all the source nodes select the same sequence of hops for their transmission. In the distributed network scenario, each non-cluster-head node is allocated a timeslot for data transmission to the destination. Analyzing the performance of such centralized and distributed network shows the dependency of source node on other nodes for transmission, high delay and low packet delivery ratio. We propose a new routing mechanism based on ant colony algorithm to average the energy consumption of main nodes and reduce the transmission time so as to give a balance of energy consumption between nodes. Thus Ant colony algorithm with centralized and distributed network can give better performance characteristics.
\end{abstract}

\section{General Terms}

Energy aware routing algorithm in MANET.

\section{Keywords}

MANET, Routing protocols, Centralized and Decentralized network, Ant Colony Optimization.

\section{INTRODUCTION}

The increasing popularity of portable devices, as an outcome of recent technological developments has realized the dream of "communication anytime and anywhere" in the present world. Mobile Adhoc NETwork (MANET) [1] is a selforganising system of mobile nodes which exhibit nomadic nature, dynamically creating and tearing down its linkages. They organise themselves in arbitrary and temporary network topologies varying with time as the nodes move or adjust their transmission and reception parameters. At any point of time, depending on the nodes' positions, their transmitter-receiver coverage patterns and transmission power levels, a purely random wireless connection - usually multi hop or "adhoc"exists between the nodes which are dynamic in nature. MANETs are characterised by [4]
1) Energy-constrained operation: Nodes in a MANET may rely on batteries or other exhaustible means for their energy. Hence the prime importance is energy optimization for such networks.

2) Dynamic topologies: The network topologies keep changing due to the free mobile nature of the nodes at unpredictable time instants.

3) Bandwidth-constrained, variable capacity links: Compared with wired connections, wireless links have lower capacity. Effects of noise, fading, multiple access and other interference conditions cause the actual throughput of wireless communications is found often very less than a radio's maximum transmission rate.

4) Limited physical security: Mobile wireless networks are generally more prone to physical security threats than our fixed wired networks. Existing link security techniques are used in wireless networks to reduce security threats. The issues related to spoofing, eavesdropping, denial of service attacks etc. should be well addressed. The decentralized nature of network control in MANETs provides additional robustness against the single point failures compared with centralized approaches

In ad hoc networks, to ensure successful delivery of a packet from sender to destination, each node must incorporate routing functionality. Such network is supposed to have dynamic, random, multi hop topologies rapidly-changing with time. Several proactive and reactive routing protocols have already emerged. Proactive routing algorithm like DSDV maintains node to node linkages beforehand maintains them by periodic transfer of control signals requiring a large control traffic handling network. Reactive protocols like AODV, DSR create paths on demand by means of flooding. But their dissemination causes a great delay in packet transmission.

Towards the aim of energy optimization many of the routing algorithms incorporate clustering approaches. The need for clustering the group of nodes arises from the fact that it makes the entire network system stable and can be managed easily. Therefore in a general network scenario a group of nodes-usually which are geographically close--combine to form a cluster with a cluster head acting as a coordinator of traffic within each cluster. The selection of this cluster head may depend on residual energy, location or any similar parameters of the node.

Some nodes may acts as relay nodes between two adjacently placed clusters and thus performs the function of a gateway. For Mobile ad hoc networks since all the nodes are mobile, in 
course of time they rearrange their positions, meanwhile transferring the head's authorities to any other satisfying the above mentioned criteria.

Clustering approaches [2] will tremendously increase packet transmission but will contribute much to delay. Therefore for increasing the data rate we go for Ant based routing protocol. The main benefits of this nature-inspired protocol include high path search capacity with commendable reduction in time delay. It is mainly implemented in the MAC layer and has far reaching applications in travelling salesman approaches.

\section{NETWORK MODEL}

The packets used for transmission in this network are mainly divided into three classes. They are data packets, forward/backward ants and neighbour control packets. [4]

The Data packets are simple IP packets which represents the information being exchanged between the two end entities. In Ant based routing algorithms the packets do not store the routing information rather it will make use of the routing table entries for the purpose of routing information between the source and destination. Forward/Backward Ants are control packets used for the updating routing table entries and to deliver the traffic load information all over the network. Apart from an IP header, it includes various data fields like Final destination address, Birth time, Arrival time and Memory stalk with the address of recently visited nodes. Neighbour Control packets are used to trace a list of nodes to which the packets can be forwarded. They are simple 'HELLO' messages broadcasted by a central node to its neighbours periodically.

Any node in a wireless network maintains an input buffer composed of a single FIFO queue and output buffer having two queues; one of low priority and the other with high priority. The queue to which the output needs to be routed is decided on the packet to me delivered and the next node maintained. The buffer size is limited and if it exceeds this limit the packets get dropped. Backward ant is supposed to have more priority compared with forward ant. The types of networks does not suggest the way in which network nodes are connected or the way that information flows (as do topologies)[6], but rather, the manner and mode in which nodes communicate with each other and share information. They are of two basic types:

\subsection{Centralized Network}

A centralized wireless network [11] is a cluster membercluster head network in which the members have no individual control; that is, all maintenance and setup occurs at the server level. In such networks all the resources are stored or managed at one place. This makes it easy for system administrator to keep all that resources consistent and in accurate form.

In such a network the central node is responsible for managing all the processing and controlling tasks. All other member nodes on the network request the services from the central node on demand. The central node is in charge to assign the rights to these different members that request the services. The extreme ease of management and ability to micromanage is a drawing point of this access method, but the prohibitive cost and inflexibility of the method deter many from employing it. As in any case such networks also have many pros and cons. Centralized server has the advantage for authentication purpose of the available resources and is strong enough to avoid hackers entering the system. Disadvantages of such system are that it requires complex computation single point failure may affect the whole network. . It means if this central device create some problem, the entire network will suffer a fall down. This central device has to handle all the clients with various requests so, it must be powerful enough to fulfil this task efficiently.

\subsection{Decentralized Network}

Often referred to as "peer to peer" network, a decentralized network does not contain any distinctions between member and head nodes. Normally in a distributed network scenario the whole system constitutes a large number of clusters; formed by grouping nodes which are located geographically close. In a decentralized network, every node can act as a member or head with time depending on the task at hand satisfying certain performance parameters. The ease of adding nodes and the ease of setup is a drawing point of decentralized networks, but the pivotal downfall of these networks is their difficulty of maintenance (a setting must be changed on each node to reflect a setting change on the whole network).

While in distributed system all the sites containing the data and resources need to be managed. We can easily back up the data that is only at one place. It is also very much easy to protect the system from authorized access because there is only site on the network that needs protections.

\section{ANTNET ALGORITHM}

Many biologically inspired routing protocols based on Swarm Intelligence techniques are now been incorporated in different areas of science which shows commendable performance enhancement. Ant based routing algorithms [3] are the most popular among them for their scalable, reliable and robust nature compared to other conventional algorithms. They are well suited in mobile network environment since they do not require any additional message exchanges to maintain the communication paths.

AntNet is an algorithm for best-effort adaptive routing in IP networks. The basic design of AntNet is based on the Ant Colony Optimization (ACO) [5], [7], [8], [9] framework, which exploits the mechanisms behind the shortest path behaviour observed in ant colonies to define a nature-inspired metaheuristic for combinatorial optimization.

In our network model, we assume that $\mathrm{N}$ number of nodes are scattered randomly over the network. These nodes are mobile in nature which keeps their location updated at each instant. The cluster head selection is based on the amount of residual energy possessed by each node after each of its transmission. The current energy [8] of each node at a particular time instant is computed as:

$$
\begin{aligned}
& \mathrm{E}_{\mathrm{CN}}=\frac{\text { Packet size } \mathrm{x} \text { Packet delivery ratio }}{\text { Rate of packet transmission }} \\
& \mathrm{E}_{\mathrm{CN}}=\frac{\mathrm{P}_{\text {size }} \times\left(\mathrm{P}_{\mathrm{R}} / \mathrm{P}_{\mathrm{T}}\right)}{\mathrm{P}_{\mathrm{r}}}
\end{aligned}
$$

where $\mathrm{E}_{\mathrm{CN}}$ is the current energy of a node at any particular time instant , $\mathrm{P}_{\text {size }}$ is the packet size (eg:800,1000..), $\mathrm{P}_{\mathrm{R}}$ and $\mathrm{P}_{\mathrm{T}}$ are received and transmitted power levels respectively in Joules. Rate of transmission is expressed in kilobytes. From the above equation we can raise the current energy or residual energy of a node by increasing the $\mathrm{P}_{\mathrm{R}} / \mathrm{P}_{\mathrm{T}}$ ratio (packet delivery ratio). 


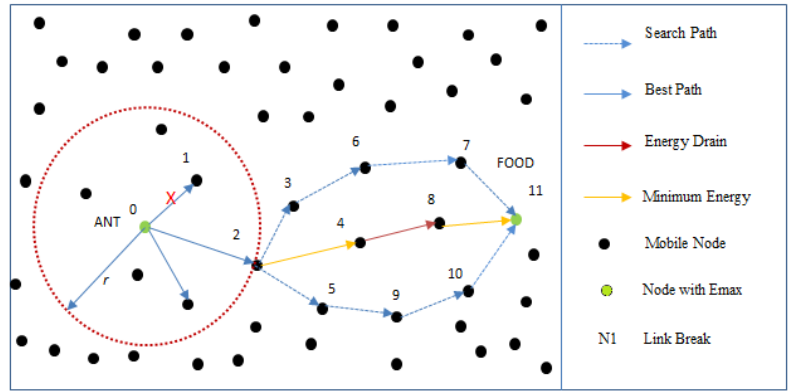

Fig 1: AntNet network scenario

In this simple scenario ant in node 0 is assumed to go in search of food at node11 through different paths. The source node 0 broadcast signals in its broadcasting range with radius ' $r$ '. Node 1, 2 are shown as neighbouring nodes of the source node. If n0-n1 linkage is broken ant will follow the path to $\mathrm{n} 2$. Hereafter it takes the strategy of multipath routing. Three different search paths are labelled in which the centre path is selected as the best path to reach the food.

\begin{tabular}{|c|c|c|}
\hline Ant & Next hop & Food \\
\hline $\mathrm{N}_{0}$ & $\mathrm{~N}_{1}, \mathrm{~N}_{2}$ & $\mathrm{~N}_{11}$ \\
\hline $\mathrm{N}_{1}$ & - & - \\
\hline $\mathrm{N}_{2}$ & $\mathrm{~N}_{4}$ & $\mathrm{~N}_{11}$ \\
\hline
\end{tabular}

If the energy possessed by a node falls below a set threshold it cannot no longer act as a cluster head and hence turn to be a member node, transferring its role to a node which satisfies the required energy criteria. As per the mentioned scenario request responses and node mobility results in energy loss in each node in a network. The energy consumption loss of the whole network can be simply formulated as:

Energy consumption loss $\left(\mathrm{E}_{\mathrm{CL}}\right)=$ Initial energy - Average energy consumed of all forwarding nodes in a particular transmission.

Let $\mathrm{E}_{\mathrm{I}}$ be the initial energy and $\mathrm{E}_{\mathrm{N}}, \mathrm{E}_{\mathrm{Nk}}, \mathrm{E}_{\mathrm{Nl}}$ etc. be the energy consumed in $\mathrm{j}^{\text {th }}, \mathrm{k}^{\text {th }}$ and $\mathrm{l}^{\text {th }}$ node respectively. Then;

$$
E_{C L}=E_{I}-\frac{\left(E_{N j}+E_{N k}+E_{N 1}+\ldots . .\right)}{n}
$$

$\mathrm{E}_{\mathrm{CL}}=\mathrm{E}_{\mathrm{I}}-\left(\Sigma \mathrm{E}_{\mathrm{Ni}} / \mathrm{n}\right) \quad$ where $\mathrm{i}=1,2,3 \ldots$

Consider a packet routing from source node to food node comprising four node hops $(n=4)$, initial energy as 100 Joules. Energy gets lost in each of the node packet transverse.

$\mathrm{E}_{\mathrm{CL}}=\mathrm{E}_{\mathrm{I}}-\frac{\left(\mathrm{E}_{\mathrm{N} 1}+\mathrm{E}_{\mathrm{N} 2}+\mathrm{E}_{\mathrm{N} 3}+\mathrm{E}_{\mathrm{N} 4}\right)}{4}$

Table 1. Energy Consumption Rates

\begin{tabular}{|l|l|l|l|l|}
\hline $\begin{array}{l}\text { Packet transmission } \\
\text { path }\end{array}$ & $\mathbf{E}_{\mathbf{N} \mathbf{1}}$ & $\mathbf{E}_{\mathbf{N} \mathbf{2}}$ & $\mathbf{E}_{\mathbf{N} \mathbf{3}}$ & $\mathbf{E}_{\mathbf{N} \mathbf{4}}$ \\
\hline Path 1 & 97 & 96 & 94 & 92 \\
\hline Path 2 & 98 & 96 & 93 & 91 \\
\hline Path 3 & 96 & 95 & 94 & 93 \\
\hline
\end{tabular}

\begin{tabular}{|r|l|l|l|l|}
\hline Path 4 & 95 & 94 & 93 & 90 \\
\hline
\end{tabular}

Fig 2: Plot for energy consumption rates for in a 4-hop multipath network

\section{PROBLEM DEFINITION}

Energy efficient packet routing with high packet delivery ratio in:

1. Centralized network

2. Decentralized network

A centralized network scenario consists of a central data collector to which the data packets from different source nodes are being routed. In a normal centralized network the data packets from different source nodes are flooded towards the central data collector. It increases the possibility of collision. This congestion results in high degree of packet loss. Hence proves to be less energy efficient.

Whereas by making use of the proposed ant based protocol, a great deal of improvement towards the aim of energy efficiency in the networks is attained. The ant algorithm can be thought of like a simple packet routing approach based on finding the one hope neighbour. It maintains a time slot or a queue to guide the packets from different source nodes to the central data collector. This effectively reduces the chances for collision, lowering the packet loss rate and thereby increasing packet delivery ratio.

A decentralized network consists of a large number of node groups forming clusters. The selection of this cluster head may depend on residual energy, location or any similar parameters of the node. For Mobile ad hoc networks since all the nodes are mobile, in course of time, they rearrange their positions, meanwhile transferring the head's authorities to any other satisfying the above mentioned criteria.

Clustering approaches will tremendously increase packet transmission but will contribute much to delay. Therefore for increasing the data rate we go for Ant based routing protocol. The main benefits of this nature-inspired protocol include high path search capacity with commendable reduction in time delay.

By in cooperating Ant technique for the packet routing purpose for both these strategies will commendably improve the packet delivery ratio by reducing the packet loss rate. An overall improvement up to $70 \%$ is obtained for both centralized and distributed ant based network scenarios. 


\section{SIMULATION RESULTS}

The proposed strategy is implemented using NS2 simulator tool. Performance analysis of AntNet protocol in centralized and decentralized network with a grid size of $1000 \times 1000 \mathrm{~m}$ is carried out. Network with 30, 50,100 nodes are considered for performance analysis. Random way point mobility model with CBR (Constant Bit Rate) traffic is used to simulate nodes movement.Important QOS parameters like delay and throughput of both centralized and decentralized network scenarios are being compared by varying the number of nodes in the network. The simulation parameters used are summarized and tabulated.

Table 2. Simulation Parameters

\begin{tabular}{|l|l|}
\hline Parameter & Value \\
\hline Number of nodes & $30,50,100$ \\
\hline Simulation Time & $20 \mathrm{~min}$ \\
\hline Packet Interval & $0.01 \mathrm{sec}$ \\
\hline Simulation Landscape & $1000 \times 1000$ \\
\hline Background Data Traffic & CBR \\
\hline Packet Size & 1000 bytes \\
\hline Initial Energy & 100 Joules \\
\hline Transmission Range & 100 Kbytes \\
\hline Node Transmission range & $250 \mathrm{~m}$ \\
\hline Antenna Type & Omni directional \\
\hline Mobility Models & Random-waypoint $(0-30 \mathrm{~m} / \mathrm{s})$ \\
\hline Routing Protocol & ANTNET \\
\hline MAC Protocol & IEEE 802.11 \\
\hline
\end{tabular}

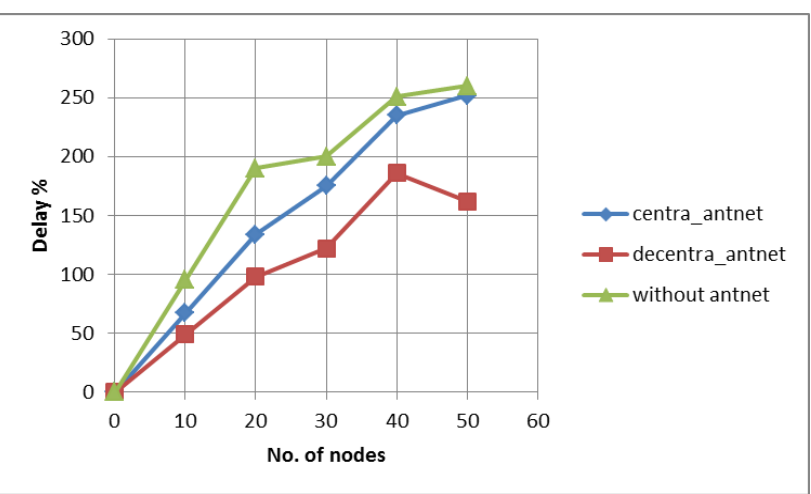

Fig 3: Comparison of delay between AntNet based centralized, decentralized network and scenario without AntNet by varying number of nodes

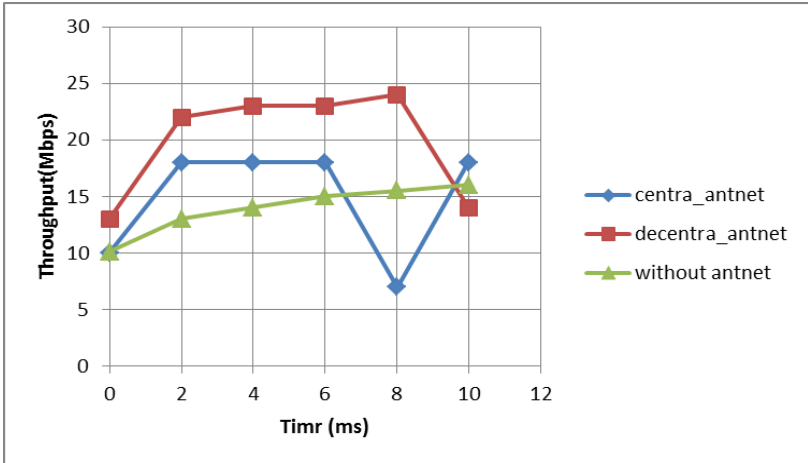

Fig 4: Comparison of throughput between AntNet based centralized, decentralized network and scenario without AntNet

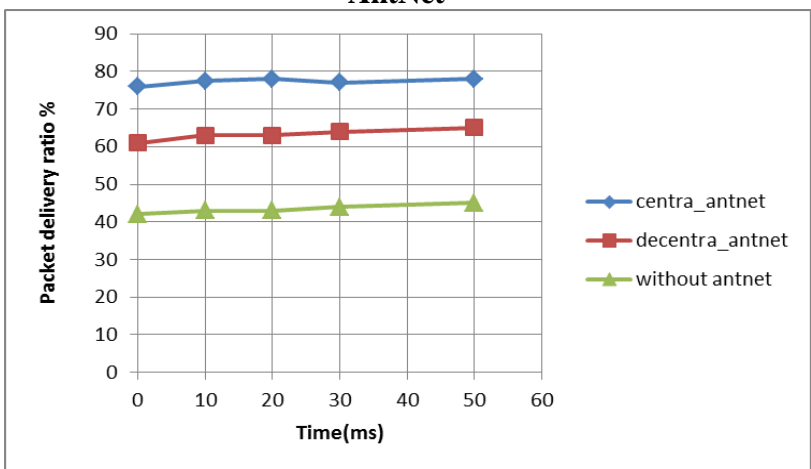

Fig 5: Comparison of Packet delivery ratio between AntNet based centralized, decentralized network and scenario without AntNet

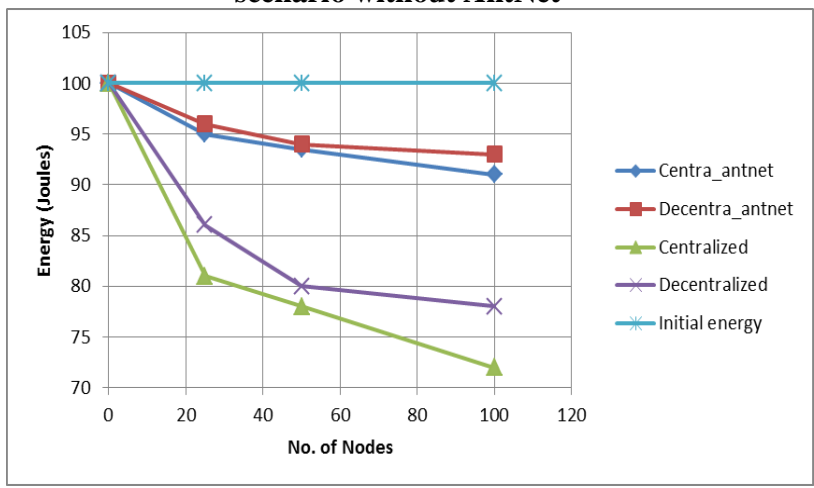

Fig 6: Comparison of Energy consumption in centralized and decentralized scenarios with and without AntNet .

Initial Energy of each node assumed to be 100 Joules.

\section{CONCLUSIONS}

A mobility based clustering algorithm based on ant colony optimization approach is proposed which resulted in commendable increase in the overall throughput and energy efficiency of a wireless network. By introducing this technique in the centralized and decentralized network scenarios the packet delivery ratio is decently increased by effectively reducing the average packet loss in a path constrained network. The various QOS parameters like delay, throughput and packet delivery ratio have been analysed in ant based centralized and decentralized scenarios with varying number of mobile nodes and compared which shown great performance improvement.

Energy consumption rate also showed tremendous improvement over the pre-existing network strategies, without the aid of ant based routing protocol of which decentralized ant net scenario gave much better result. We obtained up to 
$65 \%$ betterment compared with the networks without employing AntNet.

\section{REFERENCES}

[1] The Handbook of wireless Adhoc Network edited by Mohammad Ilyas.

[2] Handbook of Wireless Networks and Mobile Computing, Edited by Ivan Stojmenovic'.

[3] Xie Hui, Zhang Zhi-gang ,NIE Feng,A Novel Routing Protocol in Wireless Sensor Networks based on Ant Colony Optimization, International Journal of Intelligent Information Technology Application, 2010

[4] Marco Dorigoa,, Christian Blum, Ant colony optimization theory: A survey,

[5] Lianyu Wang, Qinglin Sun, Hongwen Mal, Energy Consumption Optimize Based On Ant Colony Algorithm For Wireless Sensor Networks , 2010 2nd International Asia Conference on Informatics in Control, Automation and Robotics

[6] Wenjing Guo, Wei Zhang, Gang Lu,PEGASIS Protocol in Wireless Sensor Network Based on an Improved Ant Colony Algorithm, 2010 Second International
Workshop on Education Technology and Computer Science

[7] Xiao-Min Hu and Jun Zhang, Ant Routing Optimization Algorithm for Extending the Lifetime of Wireless Sensor Networks , 2010 IEEE

[8] Wei Gao, Qinglin Sun, Zengqiang Chen ,Optimal Energy Consumption in Wireless Sensor Networks by Using the Ant Colony Algorithm(ACA) , 2010 International Conference on Computer and Communication Technologies in Agriculture Engineering

[9] Xin Guan, L. Guan , X.G. Wang, Tomoaki Ohtsuki ,A Novel Routing Algorithm based on Ant Colony System for Wireless Sensor Networks, 2009 IEEE

[10] The IEEE website. [Online]. Available: http://www.iee.org/

[11] The Handbook of wireless Adhoc Network edited by Mohammad Ilyas.

[12] Handbook of Wireless Networks and Mobile Computing, Edited by Ivan Stojmenovic 